\title{
Basal forebrain activation enhances between-trial reliability of low-frequency local field potentials (LFP) and spiking activity in tree shrew primary visual cortex (V1)
}

\author{
Paolo De Luna ${ }^{1}\left[\right.$ Julia Veit ${ }^{1,2} \cdot$ Gregor Rainer $^{1}$
}

\begin{abstract}
Brain state has profound effects on neural processing and stimulus encoding in sensory cortices. While the synchronized state is dominated by low-frequency local field potential (LFP) activity, low-frequency LFP power is suppressed in the desynchronized state, where a concurrent enhancement in gamma power is observed. Recently, it has been shown that cortical desynchronization co-occurs with enhanced between-trial reliability of spiking activity in sensory neurons, but it is currently unclear whether this effect is also evident in LFP signals. Here, we address this question by recording both spike trains and LFP in primary visual cortex during natural movie stimulation, and using isoflurane anesthesia and basal forebrain (BF) electrical activation as proxies for synchronized and desynchronized brain states. We show that indeed, low-frequency LFP modulations ("LFP events") also occur more reliably following $\mathrm{BF}$ activation. Interestingly, while being more reliable, these LFP events are smaller in amplitude compared to those generated in the synchronized brain state. We further demonstrate that differences in reliability of spiking activity between cortical states can be linked to amplitude and probability of LFP events. The correlated temporal dynamics between low-frequency LFP and spiking response reliability in visual cortex suggests that these effects may both be the result of the same neural circuit activation triggered by $\mathrm{BF}$ stimulation, which facilitates
\end{abstract}

Gregor Rainer

gregor.rainer@unifr.ch

1 Visual Cognition Laboratory, Department of Medicine, University of Fribourg, Chemin du Musée 5, 1700 Fribourg, Switzerland

2 Present Address: Department of Molecular and Cell Biology, University of California, Berkeley, CA 94720-3200, USA switching between processing of incoming sensory information in the desynchronized and reverberation of internal signals in the synchronized state.

Keywords Acetylcholine - Brain state - Gamma - Nucleus basalis $\cdot$ Synchronization/desynchronization

\section{Introduction}

The stochastic nature of neural firing is a hallmark of the cerebral cortex (Rieke et al. 1999; Shadlen and Newsome 1998; Softky and Koch 1993). In relation to sensory coding, this is demonstrated by considerable between-trial variability of spiking activity in response to an identical visual stimulus. It has become clear that brain state has a profound effect on neural response variability (Arieli et al. 1996; Azouz and Gray 1999; Kohn et al. 2009; Renart et al. 2010; Worgotter et al. 1998). The synchronized brain state, which dominates during slow wave sleep and anesthesia as well as quiet wakefulness, is characterized by synchronous fluctuations of activity in simultaneously recorded neurons (Poulet and Petersen 2008; Steriade et al. 1993) alternating with periods of network silence, in what is known as "up" and "down" states, respectively (Curto et al. 2009; Hasenstaub et al. 2007; Steriade et al. 2001). As a consequence of brain synchronization, the neural activity contains substantial shared variance unrelated to the stimulus, i.e., noise correlation (Ecker et al. 2014; Goard and Dan 2009; Poulet and Petersen 2008), while neural responses tend to be relatively poorly coupled to the sensory stimulus, which is quantified in terms of elevated Fano factor (Marguet and Harris 2011; Pinto et al. 2013) and poor between-trial reliability (Goard and Dan 2009). In the activated desynchronized state, which is associated with 
alertness and attention, noise correlations are reduced and between-trial reliability of responses is increased (Goard and Dan 2009; Marguet and Harris 2011; Pinto et al. 2013), suggesting that the cortical activity is optimized for reliable encoding of sensory information.

Brain states have distinct local field potential (LFP) signatures: low-frequency oscillations dominate during the synchronized state (Steriade et al. 1993), whereas the desynchronized state is associated with their suppression and a simultaneous enhancement of gamma-band oscillations (Harris and Thiele 2011; Lee and Dan 2012). Transitions between cortical states take place under endogenous regulation, for example, occurring during transitions from slow wave to rapid eye movement sleep, or spontaneously during quiet wakefulness (Crochet and Petersen 2006; Niell and Stryker 2008; Poulet and Petersen 2008). In particular, the basal forebrain (BF) plays a key role in regulating cortical state, such that BF activation produces both low-frequency suppression and gamma enhancement in the cortical LFP, as demonstrated by studies using electrical or optogenetic stimulation (Bhattacharyya et al. 2013; Goard and Dan 2009; Kalmbach and Waters 2014; McLin et al. 2002; Metherate et al. 1992). Converging evidence suggests that these two effects on cortical LFP may involve activation of specific BF corticopetal projection systems. Cortical gamma oscillations can be evoked by GABAergic BF projection neurons (Kim et al. 2015), while low-frequency LFP suppression tends to be sensitive to cholinergic manipulations (Chen et al. 2015; Metherate et al. 1992; Pinto et al. 2013). These latter effects probably involve not only direct BF cholinergic corticopetal projections, but also BF-mediated modulation of neural activity in thalamic nuclei (Goard and Dan 2009; Parent et al. 1988; Steriade et al. 1987).

Because cortical state affects both LFP power and between-trial reliability, the goal of this study was to investigate the relative contribution of low-frequency LFP power modulations to between-trial reliability differences between cortical states in primary visual cortex of the tree shrew, a small non-primate but highly visual mammal that is closely related to the common ancestor of placental mammals (O'Leary et al. 2013). While modulating cortical state by electrical activation of the BF, we recorded LFP and spiking responses during visual stimulation with a natural movie segment containing spatiotemporal structure that approximates what the cortex would process in realworld situations and minimizes response adaptation (Harris and Thiele 2011). We demonstrate that BF activation enhances between-trial reliability of wideband LFP signals, which is produced by differences in amplitude and occurrence probability of discrete low-frequency LFP events, whose temporal coupling with spikes accounts for enhanced between-trial reliability of spiking activity which is characteristic of the desynchronized brain state.

\section{Methods}

\section{Experimental procedures}

All experimental procedures have been performed according to local regulations approved by the veterinary office of the canton of Fribourg and in compliance with Swiss and European Union directives.

Experiments were performed in vivo on eight adult tree shrews (species Tupaia belangeri) aged 3-9 years. Preparation to surgical procedures has been previously described (Veit et al. 2011). Briefly, animals were maintained under constant anesthesia (0.5-1.5\% isoflurane in $\mathrm{O}_{2}$ ) and muscle paralysis (pancuronium-bromide i.p.) for the entire duration of the experiments. Three-to-eight data sets were acquired in each animal. During each recording session, we carefully inserted twisted-wire tetrodes in the primary visual cortex (V1) corresponding to the contralateral upper visual field representation to record neural activity (coordinates: AP $1.0 \mathrm{~mm}$, ML $4.0 \mathrm{~mm}$ ), and a $50 \mu \mathrm{m}$ diameter platinum-iridium electrode in the nucleus basalis of the basal forebrain (BF; coordinates: AP 5.9-8.0 mm, ML $1.7-3.8 \mathrm{~mm}$ ) to produce electrical micro-stimulation. $\mathrm{BF}$ stimulation was administered as a $500 \mathrm{~ms}$-long train $(50 \mu \mathrm{s}$ pulse width) of constant voltage in the range 7-10 V (at $100 \mathrm{~Hz}$ ). We sampled neural activity from the supragranular and granular layers of $\mathrm{V} 1$, based on the response to full-field drifting gratings. Then, we computed receptive field (RF) position and size by reverse correlation of spike trains with the sparse noise stimulus, as described elsewhere (Poirot et al. 2016; Ringach et al. 1997; Veit et al. 2011). Next, we analyzed the effect of BF stimulation on V1 neural activity by comparing the power spectrum of the local field potential (LFP) before and after BF stimulation. Stimulation of the BF produces cortical desynchronization, which is characterized by power decrement in the frequency range below $15 \mathrm{~Hz}$ and increment in the range 30-70 Hz. If the LFP power spectrum in V1 was desynchronized by BF stimulation, we proceeded to visually stimulate the RF with natural movie stimuli (see below). Otherwise, we advanced the stimulating electrode in steps of $50-150 \mu \mathrm{m}$ along the $z$-axis until favorable stimulating conditions were met. Multiple penetrations were made in nearby regions of $\mathrm{V} 1$, separated from each other by at least $500 \mu \mathrm{m}$. At the end of the experiment, electrolytic lesions were performed at multiple cortical depths of 2-3 previously explored recording sites. Finally, the animal was euthanized, and the brain was removed and fixed for histological inspection of recording sites. The histological 
validation of the stimulation location has been described elsewhere (Bhattacharyya et al. 2013).

\section{Visual stimuli}

Visual movie stimuli were chosen from an online database previously used for other scientific studies on the visual system (Betsch et al. 2004). We chose two movies recorded from freely roaming cats exploring the woods while wearing a head-mounted camera. Thus, visual stimuli employed in this study faithfully reproduce some of the richness of the visual environment to which animals would be normally exposed in their natural habitat, such as tree branches, leaves, grass, and often the sky. From the freelyavailable "CatCam" data set (https://zenodo.org/record/ 46481), we chose the frames 1670-1769 of movie 9 (named "labelb21ariellforest2" by the authors), and frames 2000-2099 of movie 17 ("labelmovieb000223L"). We used custom MATLAB code to extract the central $200 \times 200$-pixel region of each frame from the two sequences of movie frames, and convolved each frame with a 2D-Gaussian filter aligned to the center (standard deviation: 74 pixels) to smooth image edges. Varying Gaussian filter amplitude, we generated two stimulus contrasts: 50 and $100 \%$ of the contrast range. Then, we collated the frames in a continuous movie stimulus using the Psychophysics Toolbox for MATLAB (Brainard 1997; Kleiner et al. 2007) running on a Mac Mini. Movies were presented at 25 frames/s for $4 \mathrm{~s}$. Visual stimulation was carried out on a $21^{\prime \prime}$ cathode-ray tube computer monitor placed at $30 \mathrm{~cm}$ in front of the animal, thus subtending $\sim 60^{\circ}$ visual field. Monitor refresh rate was set to $100 \mathrm{~Hz}$ to minimize the temporal entrainment of neural activity in the primary visual cortex (V1) to the monitor refresh rate (Veit et al. 2011).

Stimuli were aligned to the center of the RF of interest, and resized to cover $5^{\circ}$ visual field regardless of RF size. Stimuli were repeated five or ten times for each contrast level, separated by $2 \mathrm{~s}$ inter-trial interval. BF electrical stimulation was administered from time -1000 to $-500 \mathrm{~ms}$ from the onset of visual stimulation.

\section{Data analysis}

We performed all the analyses described below with custom-written MATLAB code (version R2012b). Our data set consisted of 43 recording sites from which we obtained spike trains and the LFP. We focused all our analyses on the sustained response time from +500 to $+4000 \mathrm{~ms}$ relative to stimulus onset, because visual stimulus onset generates transient fluctuations in the neural activity that last for about $300 \mathrm{~ms}$ (Xing et al. 2012) and reduce between-trial response variability of V1 neurons
(Churchland et al. 2010), which could have affected our estimations of between-trial reliability. Between-trial reliability of LFP activity was computed in five frequency bands applying zero-phase bandpass filters, as follows: wideband $\quad(1-90 \mathrm{~Hz}), \quad$ low-frequency $\quad(1-10 \mathrm{~Hz}), \quad \beta$ (12-24 Hz), low- $\gamma(24-40 \mathrm{~Hz})$, and high- $\gamma(60-90 \mathrm{~Hz})$. We extracted the absolute value of the Hilbert-transformed LFP signal in each band, and computed the mean power in $100 \mathrm{~ms}-$ long non-overlapping time bins. For each stimulus movie and contrast condition, we estimated the betweentrial reliability as the mean Pearson's correlation coefficient between all unique pairs of trials $(n=10$ pairs of 5 trials, and $n=45$ pairs of 10 trials), independently for control and BF stimulation ("BFstim") conditions. We assessed statistical significance with the two-tailed Wilcoxon rank-sum test for unpaired samples and the twotailed Wilcoxon signed-rank test for paired samples. For each test, we reported the $W$ statistic and sample size, i.e., n. $P$ values were corrected for multiple comparisons with the Bonferroni method. For tests where we used nonparametric statistics, we report median and semi-interquartile range (sIQR), which is half the difference between upper and lower quartiles, alongside mean and standard error of the mean (SEM).

LFP events were found as negative peaks in the LFP amplitude timecourse, exceeding the value of mean minus 3 standard deviations (SD) of the LFP during the baseline period from -1900 to $-1200 \mathrm{~ms}$ relative to movie onset, independently for control and BFstim conditions. This threshold value was chosen to select negative LPF deflections that were readily visible by visual inspection. The amplitude distribution of selected LFP amplitudes was unimodal, because visual LFP responses evoked by the natural movie depend on the degree to which the local cortical column is activated by the preceding movie segment. Note that our results did not depend closely on the exact choice of LFP threshold, and we obtained very similar finding using lower $(2.5 \times \mathrm{SD})$ or higher $(3.5 \times \mathrm{SD})$ values. For the analyses on LFP events, we considered only recording sessions in which we could detect at least one event per stimulus repetition in the control condition. From a total of 172 recordings (i.e., 43 recording sites and 4 stimulus conditions), we identified LFP events in 42 recordings ( $25 \%$ of the total) from 15 unique recording sites spanning all stimulus conditions (medium-contrast movie 1: 13 sessions; high-contrast movie 1: 9 sessions; medium-contrast movie 2: 12 sessions; and high-contrast movie 2: 8 sessions). This subset had approximately the same number of recordings per stimulus condition ( $\chi^{2}$-test on the $2 \times 2$ contingency table of the 4 stimulus condition frequencies, $\chi^{2}=0.004, P=0.952$ ).

The LFP event count per second was computed in $50 \mathrm{~ms}-$ long bins separately for the two movies. The 
Spearman's correlation coefficient was used to assess correlation between control and BFstim event counts. The temporal alignment of LFP events on a trial-to-trial basis was computed as the minimum time between each event and any other events in all other trials. For each data set, we computed the standard deviation of a Gaussian curve fitted on these inter-LFP event times. Standard deviations were then compared in the population between the two conditions.

Spike trains were sorted with the MClust toolbox for MATLAB according to inter-spike-interval distributions and principal component analysis. For each trial, we computed the post-stimulus time histogram (PSTH) of spike trains by summing spikes in 100 ms-long non-overlapping bins. Statistical significance for each neuron was assessed with a randomization test, which was based on 1000 samples obtained by shuffling with replacement the trial-to-trial correlation coefficients and randomly reassigning these to each condition. A two-sided $P$ value was obtained by counting the number of absolute reliability values that were greater than or equal to the absolute observed reliability, divided by the number of samples plus one.

We performed firing rate (FR) equalization of spike trains to account for different neural responsivity between control and BFstim conditions. Spikes were randomly deleted from the spike trains with the greater number of spikes until responses in both conditions had equal number of spikes. This procedure was repeated 200 times, and each time we computed a correlation coefficient. We used the mean value for each neuron to calculate the between-trial reliability across the population. Randomization tests were performed for each neuron as described above.

Time-averaged Fano factors were computed for each condition as the mean ratio of variance over mean spike count in 100 ms-long non-overlapping time bins. We also computed FR-equalized Fano factors on 200 artificial spike trains per trial, obtained by randomly deleting spikes. Then, we calculated the mean Fano factor across all trials and time bins.

To examine whether spiking activity was associated with LFP events, we computed FR-equalized reliability of spiking activity after deletion of spikes that occurred either within $100 \mathrm{~ms}$ from detected LFP events or outside this interval.

The analyses on between-trial reliability of wideband LFP, bandpass-filtered LFP, and spiking activity yielded the same level of statistical significance in each animal when analyzed individually with Wilcoxon ranked-sum tests $(P<0.01)$, whose $P$ values were corrected with the Bonferroni method. Thus, we pooled the data from all animals in the results section and described the aggregated results.

\section{Results}

Electrophysiological data presented here comprised 43 recordings of both spiking activity and local field potentials (LFP) from the primary visual cortex (V1) of eight anesthetized tree shrews. Neural responses were elicited by visual stimulation with two 4 s-long sequences of natural movies, which closely approximated the visual richness and complexity to which animals would be exposed in their habitat. Note that tree shrews were housed in $6 \mathrm{~m}^{3}$ enclosures with plenty of wooden branches, hanging ropes, tunnels, and other structures. Their day-to-day visual environment was thus quite rich, contained spatial structures at different scales, and shared many characteristics of the natural movie stimuli used for visual stimulation. Each of two natural movie sequences was presented at medium $(50 \%)$ and full $(100 \%)$ contrast for five or ten repetitions to examine reliability of neural responses on a between-trial basis. Neural responses to the natural movie were recorded following electrical micro-stimulation of the basal forebrain $(\mathrm{BF})$ and in a control condition without electrical stimulation.

Reliability of LFP responses was profoundly affected by BF stimulation. This is illustrated by five LFP response trials to the same full-contrast natural movie presentation at an exemplar recording site, as shown in Fig. 1a. LFP deflections in the BF stimulation condition ("BFstim") tended to occur consistently across trials (Fig. 1a, upper panel), whereas in the control condition, the LFP contained numerous large-amplitude deflections that occurred only in single or in a minority of trials (lower panel). The decreased trial-to-trial response variability observed in the BFstim condition can be interpreted in terms of increased reliability of sensory encoding of stimulus features. For example, at the top of Fig. 1, we highlighted five stimulus frames around which a consistent negative LFP deflection occurred on all the BFstim trials, but not on all control condition trials. By averaging the correlation coefficients computed between Hilbert-transformed LFP responses binned at $100 \mathrm{~ms}$ for all trial pairs $(n=10)$, we observed that, for this exemplar site, LFP reliability for full-contrast stimuli increased from a median value of -0.09 (mean -0.01 ) in the control condition to 0.29 (mean 0.30) in the $\mathrm{BF}$ stimulation condition (Wilcoxon rank-sum test, $W_{10}=71, P=0.009$; see Fig. $1 \mathrm{~b}$ ) and from 0.05 (mean 0.02 ) to 0.27 (mean 0.30 ) for medium-contrast stimuli $\left(W_{10}=70, P=0.007\right)$. As previously demonstrated in both rodent (Metherate et al. 1992) and tree shrew (Bhattacharyya et al. 2013) sensory cortices, a second notable effect of BF stimulation on LFP response is the prominent reduction in low-frequency power and enhancement of gamma power (see Fig. 1c), often referred 


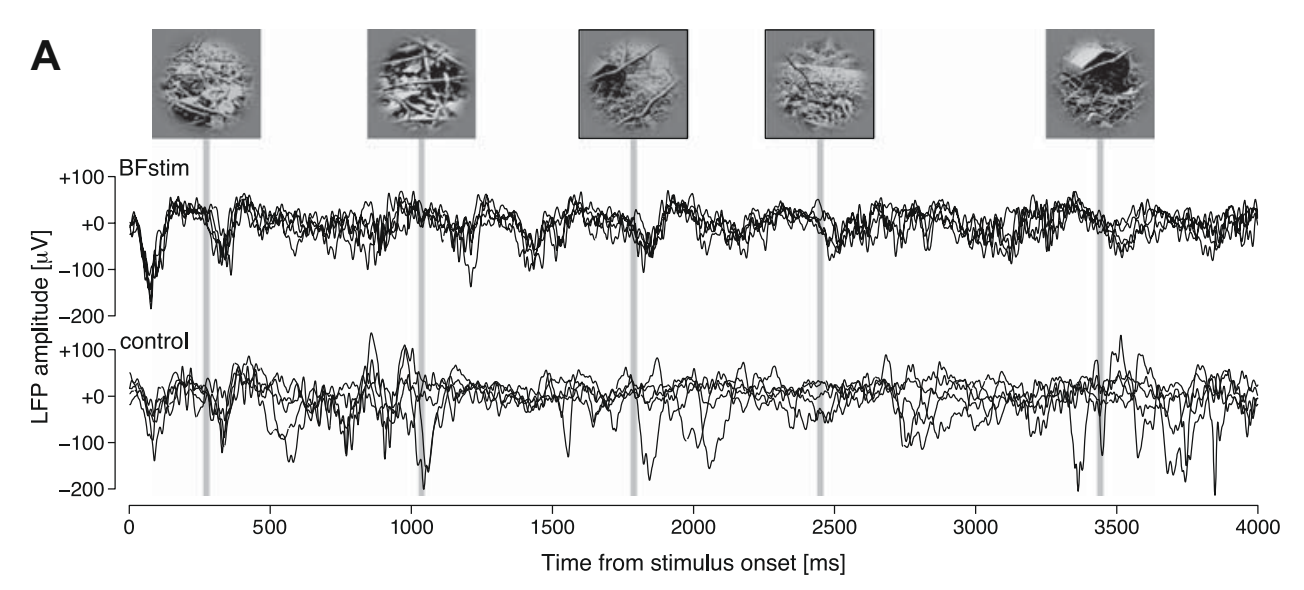



Fig. 1 Between-trial reliability of LFP activity. a Overlapping LFP traces of five trials recorded at an exemplar cortical site in response to a high-contrast natural movie stimulus during BFstim (top traces) and control (bottom traces) conditions. Five stimulus frames are shown at the top, with the time of their presentation highlighted by gray lines. b Correlation coefficients between pairs of LFP trials $(n=10$ pairs of five trials) for the same recording of a. The vertical bar marks the median value and the horizontal bar spans over median \pm sIQR.

to as cortical desynchronization. Because the notions of reliability and cortical synchronization are tightly related, in this report, we use the terms synchronization and desynchronization to refer only to modulations of cortical LFP power (see Fig. 1c), whereas increased reliability refers to reduction of trial-to-trial variability (see Fig. 1b), which produces a better temporal alignment of fluctuations across trials (see Fig. 1a).

The robust increase in LFP reliability was observable in the population of LFP recordings (Fig. 1d), in which between-trial reliability following $\mathrm{BF}$ stimulation increased from 0.06 to 0.33 for medium-contrast stimuli (median: 0.03 and 0.32 ; Wilcoxon signed-rank test, $W_{43}=14$, $P<0.001$ ) and from 0.09 to 0.33 for high-contrast stimuli (median: 0.10 and $0.31 ; W_{43}=17, P<0.001$ ). Given that LFP oscillations in particular frequency bands have been associated with cognitive processes and behaviors, we examined which LFP bands accounted for the reliability changes: low-frequency $(1-10 \mathrm{~Hz})$, beta $(12-24 \mathrm{~Hz})$, low
E



c Power spectrum for the recording of a. Vertical gray lines mark cutoff frequencies of the four bands of interest. d Between-trial reliability across the population. Each symbol represents the median reliability value of the two movie stimuli and one stimulus contrast value for each recording site. The larger symbols represent the median across recordings. e Between-trial reliability of filtered LFP for the recording of $\mathbf{a}$. Conventions follow those of $\mathbf{d}$. $* P<0.05$ for significantly increased reliability

gamma $(24-40 \mathrm{~Hz})$, and high gamma $(60-90 \mathrm{~Hz})$. We thus computed between-trial correlation coefficients for all trial pairs for appropriately bandpass-filtered LFP signals. As shown in Fig. 1e, for an exemplar recording site, LFP response reliability for high-contrast stimuli was strongly enhanced in the low-frequency and high gamma bands (Wilcoxon rank-sum test, $W_{10}=73, P=0.015$, and $W_{10}=76, P=0.029$, respectively), whereas no significant effects were observed in beta and low gamma bands $\left(W_{10}=96, \quad P=0.529 \quad\right.$ and $\quad W_{10}=85, \quad P=0.143$, respectively).

At the population level, the between-trial reliability of LFP signals following BF stimulation was most pronounced in the low-frequency and high-gamma bands (see Fig. 2a). Compared to the control condition, BF stimulation moderately enhanced response reliability in beta, low gamma, and high gamma bands (mean difference: +0.05 , +0.06 , and +0.08 respectively; median difference: +0.04 , +0.07 , and +0.09 ; Wilcoxon signed-rank test, $W_{43}=855$, 
A

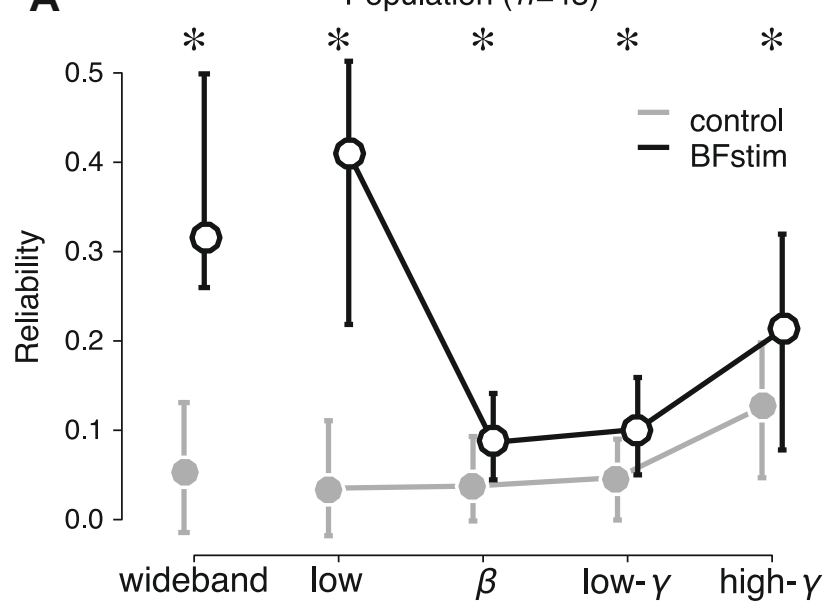

B

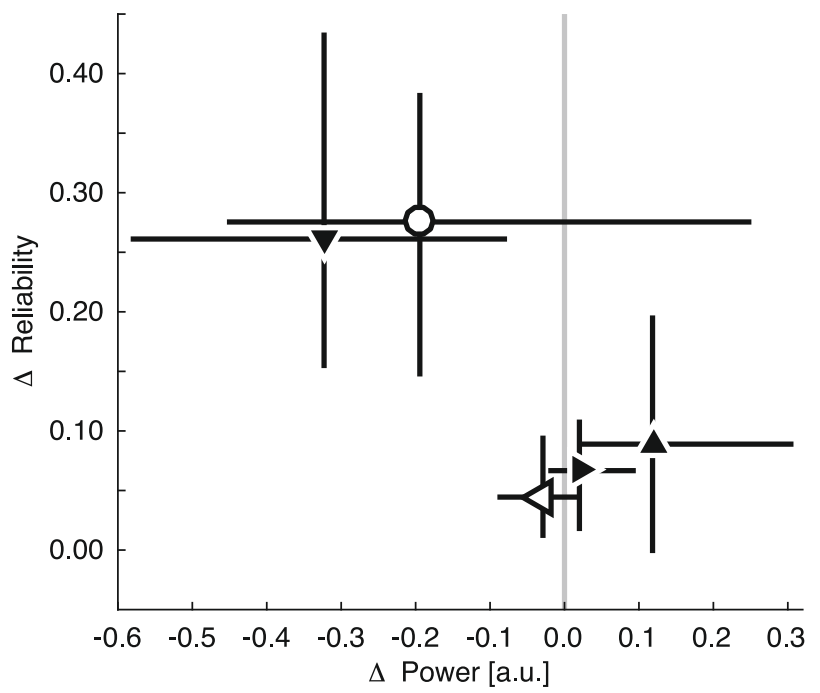

Fig. 2 Dependence of between-trial reliability and LFP power on frequency band. a Between-trial reliability of wideband (wb) and bandpass-filtered responses across all recording sites. b Variation in power and reliability of LFP responses in the wideband range and four frequency bands following BF stimulation. Symbols are: circle for wideband, downward triangle for low-frequency, leftward $\beta$, rightward low- $\gamma$, and upward high- $\gamma$. Filled symbols are for $\Delta$ power different from $0(P<0.001)$. Bars are median \pm sIQR

$W_{43}=802$, and $W_{43}=770$, respectively, $P<0.001$ for all the three bands) while producing a pronounced enhancement in the low-frequency band (mean difference: +0.28 ; median difference: $\left.+0.26 ; W_{43}=945, P<0.001\right)$. While BF stimulation thus enhanced response reliability in low-frequency and high gamma bands, it had opposite effects on LFP power in these two bands. To demonstrate this, we plotted the change in LFP band power against the change in response reliability, comparing BF stimulation to control conditions averaged across all recording sites. The results, shown in Fig. 2b, illustrate that low-frequency power was markedly reduced following BF stimulation (Wilcoxon rank-sum test, $W_{43}=154 P<0.001$ ), beta power did not systematically vary $\left(W_{43}=338\right.$, $P=0.105)$, and gamma power was moderately enhanced ( $W_{43}=700, P=0.016$ for low gamma and $W_{43}=906$, $P<0.001$ for high gamma). Despite the opposite polarity of the effects on LFP power, between-trial reliability actually increased in both low-frequency and gamma bands. Interestingly, the amount of gamma power increase and low-frequency power decrease were uncorrelated across recording sites (Spearman's correlation coefficient, $\rho=-0.06, P=0.690)$, suggesting that the two effects may result from distinct underlying mechanisms. The same was true for gamma and low-frequency reliability ( $\rho=+0.14, P=0.130)$, further consistent with multiple mechanisms involved in mediating the observed effects of BF stimulation on V1 activity.

The impact of BF stimulation on low-frequency LFP oscillations was studied in more detail, to evaluate how LFP power modulations affect response reliability to natural movie stimuli. As mentioned above, single-trial LFP responses were often dominated by large-amplitude lowfrequency negative deflections. We detected these lowfrequency deflections in the LFP independently for control and BFstim conditions, referring to them as LFP events (see Fig. 3a, cross symbols below LFP traces). Given that BF stimulation enhanced low-frequency LFP reliability, we predicted that LFP events should occur with better between-trial temporal alignment in this condition. This was indeed the case: examination of the histogram of interLFP event times for this exemplar session revealed a substantially narrower distribution in the BF stimulation compared to the control condition (SD 46 vs. $344 \mathrm{~ms}$, respectively; see Fig. 3b). This effect was significant for the population of recording sites, with median values of 86 and $298 \mathrm{~ms}$, respectively (Wilcoxon signed-rank test, $\left.W_{42}=689, P=0.002\right)$, confirming that $\mathrm{BF}$ stimulation resulted in better temporal alignment of LFP events between trials. At the same time, the amplitude of LFP events was lower following $\mathrm{BF}$ stimulation than in the control condition, both during visual stimulation (mean \pm SEM: $150 \pm 15 \mu \mathrm{V}$ and $240 \pm 18 \mu \mathrm{V}$ for BFstim and control conditions; median \pm sIQR: $158 \pm 77 \mu \mathrm{V}$ and $208 \pm 101 \mu \mathrm{V}$; Wilcoxon signed-rank test, $W_{41}=28$, $P<0.001$; see black traces in Fig. 3c) and in the absence of visual stimulation in a baseline period (mean \pm SEM: $125 \pm 15$ and $147 \pm 18 \mu \mathrm{V}$ for BFstim and control conditions; median \pm sIQR: $119 \pm 58$ and $143 \pm 92 \mu \mathrm{V}$; Wilcoxon signed-rank test, $W_{41}=473, P<0.001$; see gray traces in Fig. 3c), which explains the overall reduction in low-frequency LFP power. Considering these results in terms of signal-to-noise ratio, the LFP event amplitude $(L)$ during visual stimulation can be considered as the signal, 
Fig. 3 Characterization of LFP events. a LFP events (cross symbols) detected for the same recording site of Fig. 1a, whose traces are shown above. Left panel shows the baseline activity, in the absence of visual stimulation. b Distributions of trial-to-trial delays between each LFP event and the closest event on all other trials for the same recording of a. c Median LFP event across all 42 recordings. d Scatterplot shows mean LFP event amplitude (on the abscissa) and spiking activity (on the ordinate) for each recording during the control (gray circles) and BFstim (black circles) conditions. Lines connecting each recording's values before and after BF stimulation show LFP event amplitude tended to decrease and spiking activity tended to increase following BF stimulation. e Distribution of LFP events per unit time occurring during visual stimulation (top panel) and during the baseline period (bottom panel). f LFP event count histogram in $50 \mathrm{~ms}-$ long time bins for BFstim and control conditions during baseline (left panel) and visual stimulation (right panel). $* P<0.001$ between BFstim and control, n.s. not significant between BFstim and control
A
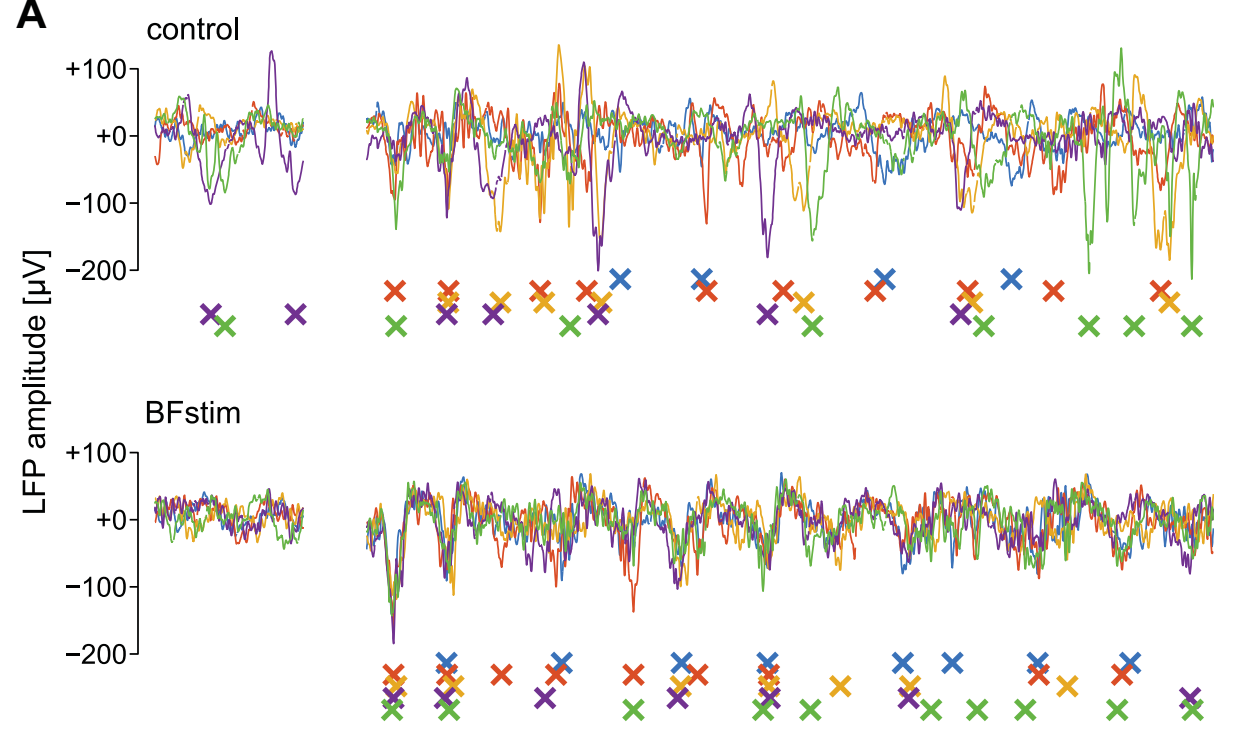

$-1900 \quad-1200$

\begin{tabular}{lllllllll}
\hline & 500 & 1000 & 1500 & 2000 & 2500 & 3000 & 3500 & 4000
\end{tabular}

B

Time from stimulus onset [ms]

D $_{100} \quad \circ$ control $\quad$ BFstim


C

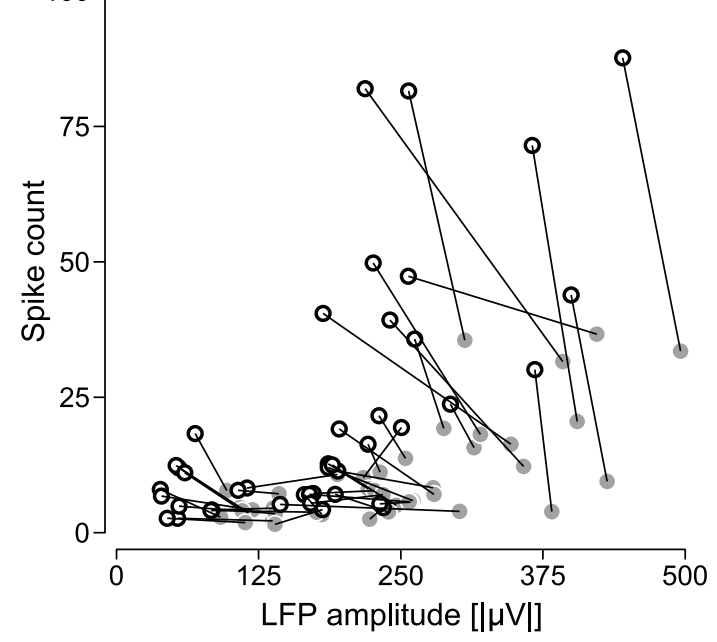

Delay [ms]

E

Visual stimulation

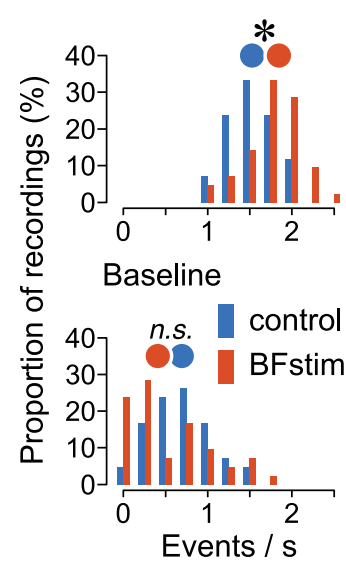

F

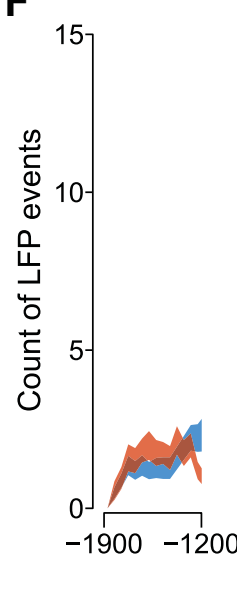

control

BFstim

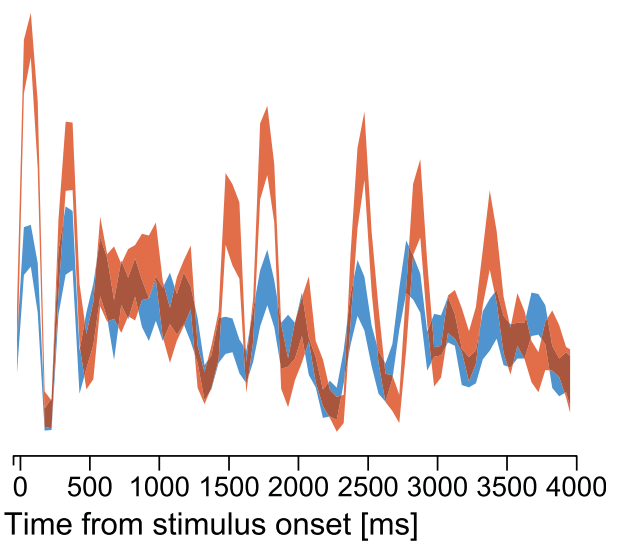


whereas a relevant measure of noise would be the variance of the LFP during the baseline period preceding visual stimulation. We found that, as expected as consequence of desynchronization, baseline variance was significantly higher in the control than BF stimulation condition ( $\sigma^{2}=0.62$ and $0.30 \mathrm{nV}^{2}$, respectively; Wilcoxon signedrank test, $\left.W_{41}=821, P<0.001\right)$. The signal-to-noise ratio $\left(\mathrm{SNR}=L / \sigma^{2}\right)$ was similar in the two conditions (mean \pm SEM: $\quad \mathrm{SNR}_{\text {control }}=3.8 \pm 0.7, \quad \mathrm{SNR}_{\mathrm{BFs}-}$ ${ }_{\text {tim }}=3.9 \pm 0.5$; median \pm sIQR: $\mathrm{SNR}_{\text {control }}=4.0 \pm 2.5$, $\mathrm{SNR}_{\mathrm{BFstim}}=4.2 \pm 2.0$; Wilcoxon signed-rank test, $\left.W_{41}=375, P=0.346\right)$. These analyses suggest that $\mathrm{BF}$ stimulation has no impact on signal-to-noise ratio of LFPs in V1, but rather impacts strongly on temporal alignment and reliability of these signals.

LFP events were associated with increased spiking activity in both conditions, but spiking activity during LFP events was more pronounced following BF stimulation than in the control condition (mean: 53 vs. 23 spikes/s; median: 22 vs 10 spikes/s; Wilcoxon signed-rank test, $\left.W_{41}=33, \quad P<0.001\right)$. Lower LFP amplitude and increased spiking activity during LFP events were observed consistently in the population of recordings, as shown in Fig. 3d. Spike count increases were more prominent for LFP amplitudes greater than $250 \mu \mathrm{V}$, which we attribute to an overall enhanced responsivity of the recorded neural population at the corresponding recording sites. However, enhancements of spike count, as well as LFP amplitude reductions, were observed across the entire range of LFP amplitudes following BF stimulation, suggesting that both populations were affected in a similar way. Given the strong relation between LFP events and spiking activity, we further characterized the occurrence frequency of LFP events. LFP events occurred frequently during visual stimulation (see Fig. 3e, upper panel), and were more numerous $(\sim 15 \%)$ following BF stimulation (mean \pm SEM, BFstim: $1.78 \pm 0.05$, control: $1.52 \pm 0.04$ events/s; median \pm sIQR, $\quad$ BFstim: $\quad 1.82 \pm 0.20$, control: $1.56 \pm 0.19$ events/s; Wilcoxon rank-sum test, $\left.W_{42}=1368, P<0.001\right)$. LFP events also occurred in the absence of visual stimulation (see Fig. 3e, lower panel) during a baseline period (mean \pm SEM, BFstim: $0.54 \pm 0.05$, control: $0.69 \pm 0.08$ events $/ \mathrm{s}$; median \pm sIQR, BFstim: $0.45 \pm 0.25$, control: $0.70 \pm 0.19$ events/s; Wilcoxon rank-sum test for median different from zero, $W_{42}=2023, P=0.001$ ), but BF stimulation did not alter the occurrence frequency of these events (Wilcoxon rank-sum test between $\mathrm{BF}$ stimulation and control, $\left.W_{42}=2024, P=0.191\right)$. The occurrence of LFP events was elevated during visual stimulus presentation compared to the baseline period (Wilcoxon rank-sum test, $W_{42}=961, P<0.001$ and $W_{42}=954, P<0.001$ for control and BFstim, respectively), suggesting that LFP events can be spontaneously generated in the absence of visual input, but their occurrence probability is related to visual activation. To illustrate the occurrence of LFP events across the duration of the natural movie, we plotted a histogram of LFP events recorded in response to an exemplar movie ( $n=21$ recorded sites). We note that LFP events tended to be time-locked to the visual stimulus in both BFstim and control conditions (see Fig. 3f, right panel), yielding a high correlation between the two conditions (Spearman's correlation coefficient, $\rho=0.58$, $P<0.001$ ). In the absence of visual stimulation (Fig. 3f, left panel), we observed no significant correlation between control and BFstim conditions (Spearman's correlation coefficient, $\rho=0.31, P=0.261$ ). Taken together, lowfrequency LFP events were accompanied by spiking activity in tree shrew V1, and these events were generated either spontaneously or as a consequence of sensory activation. BF stimulation enhanced the frequency of LFP event occurrence during visual stimulation, and producing better temporal alignment of these events from trial to trial.

To examine the relationship between enhanced LFP reliability and spiking activity, we proceeded to examine the effect of BF stimulation on spike train responses. Spike trains of an exemplar neuron in response to a movie sequence presented at full contrast during the control condition are shown in Fig. 4a (upper panel). While this neuron clearly responded to the visual stimulus, the response was highly variable between trials. For example, at around $1500 \mathrm{~ms}$, the neuron responded robustly to the visual stimulus in only two of five trials in the control condition. Computing spike train reliability similar to LFP reliability above, we estimated a mean response reliability of 0.26 for this condition. By contrast, after BF stimulation, the response reliability of the same neuron reached a value of 0.62 , and this increase was statistically significant (median: 0.26 and 0.73 , respectively; Wilcoxon rank-sum test, $\left.W_{10}=55, P<0.001\right)$. The effects observed in the exemplar neuron were representative for the population of recorded neurons. Thus, BF stimulation significantly enhanced response reliability for 26/43 neurons at $50 \%$ contrast and 29/43 neurons at $100 \%$ contrast (randomization tests corrected for multiple comparisons, $P<0.05$ ). Average between-trial reliability values were significantly increased at both contrasts, and reliability was greater for high than medium contrast in both control and BFstim conditions (Wilcoxon signed-rank test, $W_{43}=829$, $P<0.001$ and $W_{43}=840, P<0.001$ for medium and high contrast, respectively), as illustrated in Fig. $4 \mathrm{~b}$. These results in tree shrew V1 are highly similar to previous findings in rat visual cortex employing a similar dynamic stimulus (Goard and Dan 2009). Since BF stimulation 
Fig. 4 Between-trial reliability of spiking activity. a Raster plots display spiking activity of an exemplar neuron after firing rate equalization (see

"Methods"). b Distribution of between-trial reliability values of all recorded neurons.

Conventions follow those of Fig. 1d
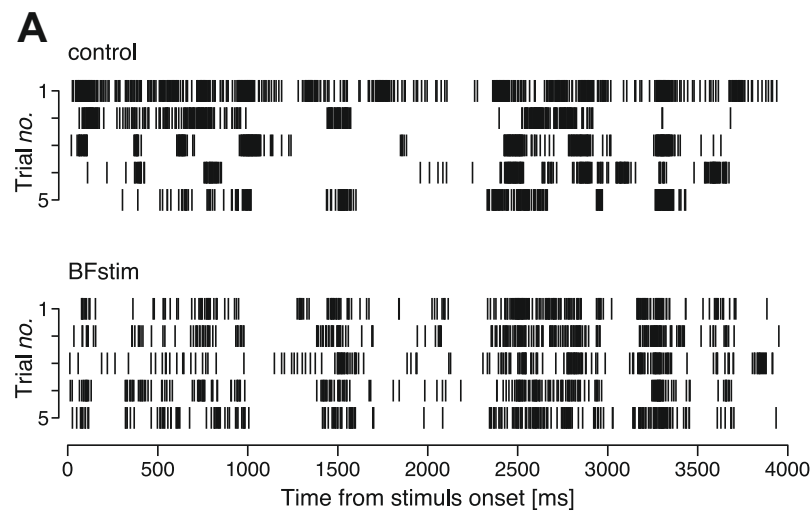

increased firing rate (FR) by on average $33 \pm 8$ spikes/s (median \pm sIQR: $9 \pm 31$; Wilcoxon signed-rank test, $\left.W_{43}=90, \quad P<0.001\right)$, we were concerned that this increase alone might account for the observed enhancement in reliability. We, therefore, repeated the reliability computation 200 times, and each time randomly deleted spikes from the spike train with higher activity until responses in the two conditions had exactly equal numbers of spikes. After FR equalization, there remained 23/43 neurons (high contrast) and 19/43 neurons (medium contrast) with significantly enhanced reliability (randomization test corrected for multiple comparisons, $P<0.05$ ). The overall enhancement in spike train reliability remained statistically significant after firing rate equalization at both contrasts (Wilcoxon signed-rank test, $W_{43}=638$, $P=0.046$ and $W_{43}=653, P=0.029$ for medium and high contrasts, respectively). To compare our findings with previous studies that estimated between-trial reliability in terms of Fano factor variations, we computed time-averaged Fano factors of spike trains in our data sets. Because the Fano factor is the ratio between variance and mean spike count, lower Fano values indicate that the spike count tended to be more similar on a trial-to-trial basis, thus being interpreted as an increase in spiking reliability. For our data set, we confirmed that BF stimulation reduced the trial-to-trial variability of neural activity in response to both medium (mean Fano factor, control 2.76 and BFstim 1.77; median Fano factor, control 2.41 and BFstim 1.59; Wilcoxon signed-rank test, $\left.W_{43}=2280, P<0.001\right)$ and high-contrast stimuli (mean control 2.75 and BFstim 1.57; median control 2.79 and BFstim 1.63; $W_{43}=2325$, $P<0.001)$. This finding remained highly statistically significant also after FR equalization (mean: from 2.35 to 1.74 for medium-contrast and from 2.35 to 1.53 for high-contrast stimuli; median: from 2.21 to 1.60 for medium-contrast and from 2.18 to 1.47 for high-contrast stimuli; Wilcoxon signed-rank test, $W_{43}=2179, P=0.007$ and $W_{43}=2320, P<0.001$ for medium and high contrast, respectively). These results obtained with different quantitative methods support the role of BF activation in enhancing between-trial reliability of spike train responses.

The above results raise the question as to what degree BF stimulation-induced modulations in between-trial reliability observed at the level of spiking activity relate to changes in the LFP. In particular, we hypothesized that low-frequency events might be closely associated with the spiking reliability increases, because LFP reliability was most strongly enhanced at low frequencies. To address this issue, we randomly deleted spikes from spike trains either around or far from LFP events (see Fig. 5a), and compared the result of this manipulation with the original spiking reliability values, as shown in Fig. 5b. The result of the simulation deleting spikes within $100 \mathrm{~ms}$ of LFP events across recording sites is shown in Fig. 5c. Spiking associated with LFP events did indeed contribute significantly to between-trial reliability of spiking responses: whereas $\mathrm{BF}$ stimulation enhanced between-trial reliability for the original unperturbed spike trains (mean \pm SEM: from $0.14 \pm 0.02$ for control to $0.18 \pm 0.02$ for BFstim; median \pm sIQR: from $0.13 \pm 0.10$ for control to $0.17 \pm 0.08$ for BFstim; Wilcoxon signed-rank test, $W_{42}=310$, $P=0.049$; see Fig. 5b), it had no such effect if spikes around LFP events were deleted (mean \pm SEM: from $0.10 \pm 0.01$ for control to $0.11 \pm 0.01$ for BFstim; median \pm sIQR: from $0.08 \pm 0.07$ for control to $0.09 \pm 0.04$ for BFstim; $W_{42}=397, P=0.504$; see Fig. $5 \mathrm{c}$ ). This observation suggests that $\mathrm{BF}$ stimulation-induced modifications in LFP event temporal distribution are in fact closely related to enhanced between-trial reliability of spiking activity. To verify this, we also randomly deleted spikes occurring outside the interval from -100 to $+100 \mathrm{~ms}$ around detected LFP events, and recomputed the betweentrial reliability values (see Fig. 5d). The histogram of between-trial reliability showed that $\mathrm{BF}$ stimulation induced an enhancement in spiking reliability despite ignoring spikes not occurring during LFP events (mean \pm SEM: from $0.05 \pm 0.02$ for control to $0.08 \pm 0.01$ for BFstim; median \pm sIQR: from 

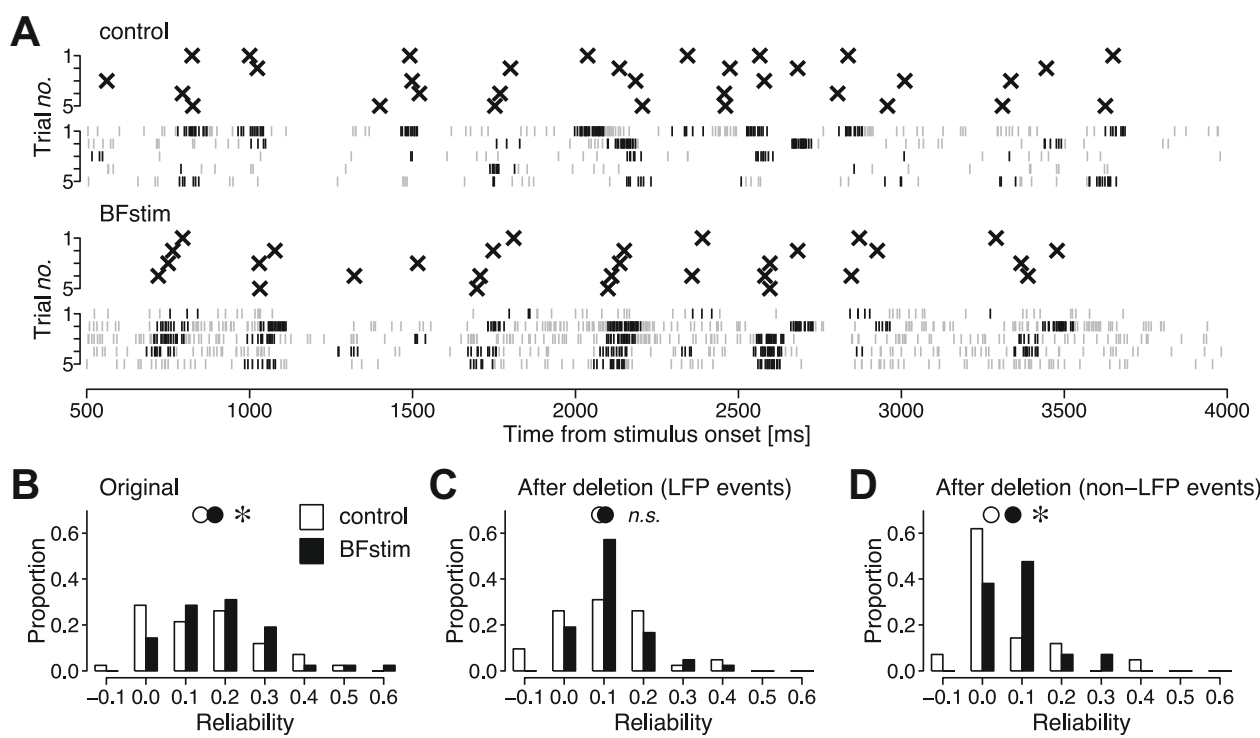

Fig. 5 Between-trial spiking reliability associated with LFP events. a Detected LFP events and corresponding spike trains for an exemplar recording. Spikes within $100 \mathrm{~ms}$ from an LFP event are highlighted in black. In two subsequent analyses, spikes close to (in black) or occurring far from an LFP event (in gray) were randomly deleted. b Histograms of between-trial spiking reliability for control and BFstim conditions before deletion of spikes. c Histograms of

$0.02 \pm 0.06$ for control to $0.07 \pm 0.04$ for BFstim; $W_{42}=269, \quad P=0.022$; see Fig. $5 \mathrm{~d}$ ). This effect was smaller in magnitude compared to the unperturbed spike train condition (mean \pm SEM: $0.05 \pm 0.02$ for control and $0.08 \pm 0.01$ for BFstim; median \pm sIQR: $0.02 \pm 0.06$ for control and $0.07 \pm 0.04$ for BFstim; Wilcoxon signed-rank test between original and after deletion during non-LFP events, $W_{42}=835, P<0.001$ for control and $W_{42}=786$, $P<0.001$ for BFstim). However, the enhancement in between-trial reliability following $\mathrm{BF}$ stimulation remained unaffected after deletion of spikes not associated with LFP events (mean \pm SEM reliability difference after spike deletion compared to original: $-0.09 \pm 0.01$ for control and $-0.10 \pm 0.02$ for BFstim; median $\pm \mathrm{sIQR}$ : $-0.07 \pm 0.07$ for control and $-0.08 \pm 0.09$ for BFstim; Wilcoxon signed-rank test, $W_{42}=467, P=0.853$ ). This analysis demonstrated a close link between low-frequency LFP modulations and changes in the reliability of spiking activity in primary visual cortex following BF stimulation.

\section{Discussion}

The main finding of this study is that BF stimulation enhanced trial-to-trial reliability of LFP signals and that this reliability enhancement was most prominent in the low-frequency band of the LFP. Interestingly, while the LFPs in this band were more reliable following $\mathrm{BF}$ between-trial spiking reliability after deletion of spikes occurring within $100 \mathrm{~ms}$ from an LFP event (spikes in black in the example of a). d Histograms of between-trial spiking reliability after deletion of spikes occurring outside the interval from -100 to $+100 \mathrm{~ms}$ from an LFP event (spikes in gray in the example of a). ${ }^{*} P<0.05$, n.s. not significant

stimulation, they were at the same time lower in amplitude compared to the control condition. We note that this finding is incompatible with the idea that BF stimulation enhances signal-to-noise ratio (SNR) of neural responses, and in fact, our analyses suggest that the SNR is not impacted by BF stimulation. In absolute terms, however, low-frequency LFP deflections were smaller in amplitude and yet more reliably evoked from trial to trial. This effect was peculiar to the low-frequency LFP. For the high gamma band, where reliability was also enhanced following BF stimulation, these enhancements were accompanied by increases in LFP power. Thus, the gamma-band LFP behaves similar to spiking activity in that both activity and reliability are increased concomitantly (Bhattacharyya et al. 2013; Goard and Dan 2009; Pinto et al. 2013). Interestingly, our analyses reveal that high-gamma and low-frequency band power modulations were uncorrelated across recording sites, suggesting that the effects reported here are due to multiple contributing BF projection systems. Taken together, BF stimulation tends to enhance excitability and responsivity of $\mathrm{V} 1$ neurons, leading to elevated spiking activity. Under these conditions, neural activity is triggered more reliably by sensory activation. However, BF activation exerts its effects on large cortical populations with diverse tuning properties. As a consequence, neighboring neurons may respond at additional, different times to the natural movie after BF stimulation, leading to temporally and spatially more distributed 
population activity that may be the reason for the reduced amplitude of the low-frequency-evoked LFP responses in this condition.

LFP power modulations have been previously linked to cortical state, such that the desynchronized state is associated with a reduction in low-frequency power in LFP and EEG signals (Bhattacharyya et al. 2013; Buzsaki et al. 1988; Chen et al. 2015; Fuller et al. 2011; McLin et al. 2002; Metherate et al. 1992; Pinto et al. 2013). Low-frequency LFP power reduction has been linked to a general suppression in depth-negative waves, which are large-amplitude low-frequency LFP events that occur spontaneously during anesthesia or quiet wakefulness (Harris and Thiele 2011) in the synchronized state. These depth-negative waves are thought to be results of spontaneous fluctuations of neural populations (Arieli et al. 1996; Han et al. 2008) through recurrent and lateral projections, leading to strong coordinated spiking discharge and large-amplitude evoked LFP responses. Prominent lateral excitatory connections have indeed been documented in tree shrew V1, where they are thought to contribute to elaboration of orientation selectivity (Chisum et al. 2003; Van Hooser et al. 2013). Here, LFP events in the desynchronized state were considerably lower in amplitude both during and in the absence of V1 visual stimulation. This lower amplitude is consistent with, and likely a consequence of, muscarinic acetylcholine $(\mathrm{ACh})$ receptor $(\mathrm{mAChR})$ activation following $\mathrm{BF}$ stimulation, because cortical desynchronization is known to be blocked by muscarinic antagonists (Goard and Dan 2009; Metherate et al. 1992; Munk et al. 1996) and the strength of intracortical synapses is depressed by mAChR activation (Gil et al. 1997; Hsieh et al. 2000). In the current experiments, nAChR currents are unlikely to contribute much to the observed findings, because the corresponding ionic transmembrane currents are strongly suppressed by isoflurane anesthesia (Campagna et al. 2003; Violet et al. 1997). The situation differs from previous studies that employed urethane anesthesia (Goard and Dan 2009; Marguet and Harris 2011), which moderately enhances nAChR ionic currents (Haensel et al. 2015; Hara and Harris 2002). In addition, while we have observed lower LFP event amplitude in the desynchronized state here, the opposite would occur with nicotinic receptor (nAChR) activation, which is expected to enhance LFP amplitudes by activating thalamocortical glutamatergic afferents (Disney et al. 2007; Gil et al. 1997; Kang and Vaucher 2009) and disinhibiting pyramidal cells through GABAergic neurons (Christophe et al. 2002). These observations suggest that cortical nAChRs likely only play a lesser role in regulating cortical responses in the present experiments than mAChR-mediated effects.

Our findings of enhanced reliability of LFP and spiking signals are closely related to work on noise correlation in visual cortex, which examines shared variability between neurons in response to visual stimulation. In fact, high noise correlation in the synchronized state is related to substantial redundant information in the neural responses, which negatively affects the decoding capability of the network (Bair et al. 2001; Ecker et al. 2010; Hansen et al. 2012; Mitchell et al. 2009; Shadlen and Newsome 1998; Sompolinsky et al. 2001; Zohary et al. 1994). We suggest that the poor between-trial reliability observed in our study is a reflection of coordinated bursts of neural activity resulting from spontaneous activity fluctuations, and accordingly cortical desynchronization both reduces noise correlation among neurons (Chen et al. 2015; Goard and Dan 2009; Pinto et al. 2013), and enhances reliability between trials.

We show that enhanced reliability in the desynchronized brain state occurs also at the level of spiking activity. This observation in the tree shrew directly parallels previous reports in the rodent visual (Goard and Dan 2009; Pinto et al. 2013) and auditory cortices (Marguet and Harris 2011) in the desynchronized state, showing that enhanced spiking reliability following $\mathrm{BF}$ activation is conserved among rodent species and tree shrew, whose primary visual cortex shares many features with that of primates (Veit et al. 2011). Our findings also show that elevated reliability of spiking activity following BF activation is accompanied by enhancements in firing rate, which, however, cannot alone account for the increased reliability as we demonstrate in control analyses with spike count normalization. The fact that both firing rate and reliability are enhanced is of interest, because activation of GABAergic BF corticopetal neurons has been shown to produce elevated cortical gamma oscillations (Kim et al. 2015), providing a separate non-cholinergic pathway by which the BF could regulate cortical activity. Indeed, we have previously argued that increases in firing rate and contrast sensitivity of visual cortex neurons likely involve BF GABAergic activation (Bhattacharyya et al. 2013). It is thus likely that GABAergic BF projections contribute to the effects reported here. In addition, it is probable that GABAergic cortical interneurons also contribute to reliability regulation, based on recent results showing that suppression of parvalbumin GABAergic interneuron activity in rodent V1 decreases response reliability (Zhu et al. 2015), and pharmacological GABA blockade in macaque extrastriate visual cortex enhances Fano factors (Thiele et al. 2012). Our findings thus argue for an involvement of both cortical and BF GABAergic cells, as well as an important cholinergic contribution to reliability effects, which is most likely mediated by M2 muscarinic ACh receptors. These are highly concentrated on cortical GABAergic dendrites (Erisir et al. 2001), providing a potential mechanism for $\mathrm{ACh}$ to regulate cortical interneuron activity and enhance 
response reliability. An alternative explanation for enhanced V1 reliability involves feed-forward effects from the thalamic lateral geniculate nucleus (LGN), as a result of $\mathrm{BF}$ projections to the reticular nucleus of the thalamus, as previously discussed (Thiele et al. 2012). In fact, it has been observed that spiking reliability in the LGN is also enhanced following BF electrical stimulation (Goard and Dan 2009). However, this effect could also be mediated by V1 infragranular layer neurons, which express mAChR (Disney and Aoki 2008; Disney et al. 2006) and are the main source of LGN top-down modulatory inputs (Fitzpatrick et al. 1994). It thus remains possible that the LGN effects are a consequence and not the cause of V1 reliability modulations following BF stimulation. However, $\mathrm{mAChR}$ blockade does not appear to attenuate $\mathrm{V} 1$ response reliability (Goard and Dan 2009), and ACh application has little impact on visual cortex Fano factors (Thiele et al. 2012), arguing for a contribution of indirect BF effects on V1 involving thalamic relay centers. Further experiments are needed to disentangle the various effects on cortical responsivity, sensitivity, and variability associated with transitions between brain states. Our findings suggest that the aspects of low-frequency LFP events may be a sensitive indicator for studying these brain state-dependent differences in sensory processing.

In conclusion, our findings suggest that tracking lowfrequency LFP event signals, and in particular, their between-trial reliability, may provide a sensitive method for determining brain state-dependent encoding of sensory signals at the level of spiking activity. In addition, due to the apparent close involvement of the cholinergic system in these effects, reduction in response reliability of sensory information may serve as early indicator of dysfunctional cholinergic neuromodulation, which can occur during certain forms of dementia (Ray et al. 2008; Siegel and Konig 2003; Sirota et al. 2008). Furthermore, visual hallucinations, which are commonly observed during Lewy body dementia and schizophrenia (Engel et al. 2001), may be related to the reliability effects reported here, such that brain state tends to sporadically synchronize, leading to depth-negative wave activity that is erroneously interpreted as visual input by cortical circuits. Tracking not the amplitude, but instead, the reliability of evoked potentials in human EEG signals over sensory cortices could thus provide a hitherto little explored biomarker for neurodegenerative processes involving the cholinergic system.

Acknowledgements This work was supported by the Schweizerische Nationalfonds (Grant Number 143390, to GR), the EURYI program (to GR), and the University of Fribourg. We thank M. Harvey and S. Sachidhanandam for helpful comments on the manuscript.

\section{Compliance with ethical standards}

Conflict of interest The authors declare no competing financial interests.

\section{References}

Arieli A, Sterkin A, Grinvald A, Aertsen A (1996) Dynamics of ongoing activity: explanation of the large variability in evoked cortical responses. Science 273:1868-1871. doi:10.1126/science. 273.5283.1868

Azouz R, Gray CM (1999) Cellular mechanisms contributing to response variability of cortical neurons in vivo. J Neurosci 19:2209-2223

Bair W, Zohary E, Newsome WT (2001) Correlated firing in macaque visual area MT: time scales and relationship to behavior. J Neurosci 21:1676-1697

Betsch BY, Einhauser W, Kording KP, Konig P (2004) The world from a cat's perspective-statistics of natural videos. Biol Cybern 90:41-50. doi:10.1007/s00422-003-0434-6

Bhattacharyya A, Veit J, Kretz R, Bondar I, Rainer G (2013) Basal forebrain activation controls contrast sensitivity in primary visual cortex. BMC Neurosci 14:55. doi:10.1186/1471-2202-1455

Brainard DH (1997) The psychophysics toolbox. Spatial Vis 10:433-436

Buzsaki G, Bickford RG, Ponomareff G, Thal LJ, Mandel R, Gage FH (1988) Nucleus basalis and thalamic control of neocortical activity in the freely moving rat. J Neurosci 8:4007-4026

Campagna JA, Miller KW, Forman SA (2003) Drug therapy: mechanisms of actions of inhaled anesthetics. N Engl J Med 348:2110-2124

Chen NY, Sugihara H, Sur M (2015) An acetylcholine-activated microcircuit drives temporal dynamics of cortical activity. Nat Neurosci 18:892-U340. doi:10.1038/nn.4002

Chisum HJ, Mooser F, Fitzpatrick D (2003) Emergent properties of layer $2 / 3$ neurons reflect the collinear arrangement of horizontal connections in tree shrew visual cortex. J Neurosci 23:2947-2960 (pii: 23/7/2947)

Christophe E, Roebuck A, Staiger JF, Lavery DJ, Charpak S, Audinat E (2002) Two types of nicotinic receptors mediate an excitation of neocortical layer I interneurons. J Neurophysiol 88:1318-1327. doi:10.1152/jn.00199.2002

Churchland MM et al (2010) Stimulus onset quenches neural variability: a widespread cortical phenomenon. Nat Neurosci 13:369-U325. doi:10.1038/nn.2501

Crochet S, Petersen CCH (2006) Correlating whisker behavior with membrane potential in barrel cortex of awake mice. Nat Neurosci 9:608-610. doi:10.1038/nn1690

Curto C, Sakata S, Marguet S, Itskov V, Harris KD (2009) A simple model of cortical dynamics explains variability and state dependence of sensory responses in urethane-anesthetized auditory cortex. J Neurosci 29:10600-10612. doi:10.1523/Jneurosci. 2053-09.2009

Disney AA, Aoki C (2008) Muscarinic acetylcholine receptors in macaque V1 are most frequently expressed by parvalbuminimmunoreactive neurons. J Comp Neurol 507:1748-1762. doi:10.1002/cne.21616

Disney AA, Domakonda KV, Aoki C (2006) Differential expression of muscarinic acetylcholine receptors across excitatory and inhibitory cells in visual cortical areas V1 and V2 of the macaque monkey. J Comp Neurol 499:49-63. doi:10.1002/cne. 21096 
Disney AA, Aoki C, Hawken MJ (2007) Gain modulation by nicotine in macaque v1. Neuron 56:701-713. doi:10.1016/j.neuron.2007. 09.034

Ecker AS, Berens P, Keliris GA, Bethge M, Logothetis NK, Tolias AS (2010) Decorrelated neuronal firing in cortical microcircuits. Science 327:584-587. doi:10.1126/science. 1179867

Ecker AS et al (2014) State dependence of noise correlations in macaque primary visual cortex. Neuron 82:235-248. doi:10. 1016/j.neuron.2014.02.006

Engel AK, Fries P, Singer W (2001) Dynamic predictions: oscillations and synchrony in top-down processing. Nat Rev Neurosci 2:704-716. doi:10.1038/35094565

Erisir A, Levey AI, Aoki C (2001) Muscarinic receptor M-2 in cat visual cortex: laminar distribution, relationship to gammaaminobutyric acidergic neurons, and effect of cingulate lesions. J Comp Neurol 441:168-185. doi:10.1002/Cne.1405

Fitzpatrick D, Usrey WM, Schofield BR, Einstein G (1994) The sublaminar organization of corticogeniculate neurons in layer 6 of macaque striate cortex. Vis Neurosci 11:307-315

Fuller P, Sherman D, Pedersen NP, Saper CB, Lu J (2011) Reassessment of the structural basis of the ascending arousal system. J Comp Neurol 519:933-956. doi:10.1002/cne.22559

Gil Z, Connors BW, Amitai Y (1997) Differential regulation of neocortical synapses by neuromodulators and activity. Neuron 19:679-686 (pii: S0896-6273(00)80380-3)

Goard M, Dan Y (2009) Basal forebrain activation enhances cortical coding of natural scenes. Nat Neurosci 12:1444-1449. doi:10. 1038/nn.2402

Haensel JX, Spain A, Martin C (2015) A systematic review of physiological methods in rodent pharmacological MRI studies. Psychopharmacology 232:489-499. doi:10.1007/s00213-0143855-0

Han F, Caporale N, Dan Y (2008) Reverberation of recent visual experience in spontaneous cortical waves. Neuron 60:321-327. doi:10.1016/j.neuron.2008.08.026

Hansen BJ, Chelaru MI, Dragoi V (2012) Correlated variability in laminar cortical circuits. Neuron 76:590-602. doi:10.1016/j. neuron.2012.08.029

Hara K, Harris RA (2002) The anesthetic mechanism of urethane: the effects on rotransmitter-gated ion channels. Anesth Analg 94:313-318. doi:10.1097/00000539-200202000-00015

Harris KD, Thiele A (2011) Cortical state and attention. Nat Rev Neurosci 12:509-523. doi:10.1038/nrn3084

Hasenstaub A, Sachdev RNS, McCormick DA (2007) State changes rapidly modulate cortical neuronal responsiveness. J Neurosci 27:9607-9622. doi:10.1523/Jneurosci.2184-07.2007

Hsieh CY, Cruikshank SJ, Metherate R (2000) Differential modulation of auditory thalamocortical and intracortical synaptic transmission by cholinergic agonist. Brain Res 880:51-64 (pii: S0006-8993(00)02766-9)

Kalmbach A, Waters J (2014) Modulation of high- and low-frequency components of the cortical local field potential via nicotinic and muscarinic acetylcholine receptors in anesthetized mice. J Neurophysiol 111:258-272. doi:10.1152/jn.00244.2013

Kang JI, Vaucher E (2009) Cholinergic pairing with visual activation results in long-term enhancement of visual evoked potentials. PLoS One 4:e5995. doi:10.1371/journal.pone.0005995

Kim $\mathrm{T}$ et al (2015) Cortically projecting basal forebrain parvalbumin neurons regulate cortical gamma band oscillations (vol 112, pg 3535. P Natl Acad Sci USA 112:E2848-E2848. doi:10.1073/ pnas. 1507465112

Kleiner M, Brainard D, Pelli D (2007) What's new in psychtoolbox3? In: Abstract supplement of the 30th European conference on visual perception (ECVP), vol 36, p 14
Kohn A, Zandvakili A, Smith MA (2009) Correlations and brain states: from electrophysiology to functional imaging. Curr Opin Neurobiol 19:434-438. doi:10.1016/j.conb.2009.06.007

Lee SH, Dan Y (2012) Neuromodulation of brain states. Neuron 76:209-222. doi:10.1016/j.neuron.2012.09.012

Marguet SL, Harris KD (2011) State-dependent representation of amplitude-modulated noise stimuli in rat auditory cortex. J Neurosci 31:6414-6420. doi:10.1523/Jneurosci.5773-10.2011

McLin DE 3rd, Miasnikov AA, Weinberger NM (2002) Induction of behavioral associative memory by stimulation of the nucleus basalis. Proc Natl Acad Sci USA 99:4002-4007. doi:10.1073/ pnas.062057099

Metherate R, Cox CL, Ashe JH (1992) Cellular bases of neocortical activation: modulation of neural oscillations by the nucleus basalis and endogenous acetylcholine. J Neurosci 12:4701-4711

Mitchell JF, Sundberg KA, Reynolds JH (2009) Spatial attention decorrelates intrinsic activity fluctuations in macaque area V4. Neuron 63:879-888. doi:10.1016/j.neuron.2009.09.013

Munk AA, Adjemian RA, Zhao J, Ogbaghebriel A, Shrier A (1996) Electrophysiological properties of morphologically distinct cells isolated from the rabbit atrioventricular node. J Physiol-Lond 493:801-818

Niell CM, Stryker MP (2008) Highly selective receptive fields in mouse visual cortex. J Neurosci 28:7520-7536. doi:10.1523/ JNEUROSCI.0623-08.2008

O'Leary MA et al (2013) The placental mammal ancestor and the post-K-Pg radiation of placentals. Science 339:662-667. doi:10. 1126/science. 1229237

Parent A, Pare D, Smith Y, Steriade M (1988) Basal forebrain cholinergic and noncholinergic projections to the thalamus and brain-stem in cats and monkeys. J Comp Neurol 277:281-301. doi:10.1002/cne. 902770209

Pinto L et al (2013) Fast modulation of visual perception by basal forebrain cholinergic neurons. Nat Neurosci 16:1857-1863. doi: $10.1038 / \mathrm{nn} .3552$

Poirot J, De Luna P, Rainer G (2016) Neural coding of image structure and contrast polarity of Cartesian, hyperbolic, and polar gratings in the primary and secondary visual cortex of the tree shrew. J Neurophysiol 115:2000-2013. doi:10.1152/jn.01000. 2015

Poulet JFA, Petersen CCH (2008) Internal brain state regulates membrane potential synchrony in barrel cortex of behaving mice. Nature 454:881-U836. doi:10.1038/nature07150

Ray S, Hsiao SS, Crone NE, Franaszczuk PJ, Niebur E (2008) Effect of stimulus intensity on the spike-local field potential relationship in the secondary somatosensory cortex. J Neurosci 28:7334-7343. doi:10.1523/JNEUROSCI.1588-08.2008

Renart A, de la Rocha J, Bartho P, Hollender L, Parga N, Reyes A, Harris KD (2010) The asynchronous state in cortical circuits. Science 327:587-590. doi:10.1126/science.1179850

Rieke F, Warland D, Steveninck RDRV, Bialek W (1999) Spikes: exploring the neural code. MIT Press, Cambridge. https:// mitpress.mit.edu/books/spikes

Ringach DL, Sapiro G, Shapley R (1997) A subspace reversecorrelation technique for the study of visual neurons. Vision Res 37:2455-2464 (pii: S0042-6989(96)00247-7)

Shadlen MN, Newsome WT (1998) The variable discharge of cortical neurons: implications for connectivity, computation, and information coding. J Neurosci 18:3870-3896

Siegel M, Konig P (2003) A functional gamma-band defined by stimulus-dependent synchronization in area 18 of awake behaving cats. J Neurosci 23:4251-4260

Sirota A, Montgomery S, Fujisawa S, Isomura Y, Zugaro M, Buzsaki $\mathrm{G}$ (2008) Entrainment of neocortical neurons and gamma 
oscillations by the hippocampal theta rhythm. Neuron 60:683-697. doi:10.1016/j.neuron.2008.09.014

Softky WR, Koch C (1993) The highly irregular firing of corticalcells is inconsistent with temporal integration of random EPSPS. J Neurosci 13:334-350

Sompolinsky H, Yoon H, Kang KJ, Shamir M (2001) Population coding in neuronal systems with correlated noise. Phys Rev E. doi:10.1103/Physreve.64.051904

Steriade M, Parent A, Pare D, Smith Y (1987) Cholinergic and noncholinergic neurons of cat basal forebrain project to reticular and mediodorsal thalamic nuclei. Brain Res 408:372-376. doi:10.1016/0006-8993(87)90408-2

Steriade M, Mccormick DA, Sejnowski TJ (1993) Thalamocortical oscillations in the sleeping and aroused brain. Science 262:679-685. doi:10.1126/science.8235588

Steriade M, Timofeev I, Grenier F (2001) Natural waking and sleep states: a view from inside neocortical neurons. J Neurophysiol 85:1969-1985

Thiele A, Herrero JL, Distler C, Hoffmann KP (2012) Contribution of cholinergic and GABAergic mechanisms to direction tuning, discriminability, response reliability, and neuronal rate correlations in macaque middle temporal area. J Neurosci 32:16602-16615. doi:10.1523/Jneurosci.0554-12.2012

Van Hooser SD, Roy A, Rhodes HJ, Culp JH, Fitzpatrick D (2013) Transformation of receptive field properties from lateral geniculate nucleus to superficial V1 in the tree shrew. J Neurosci 33:11494-11505. doi:10.1523/Jneurosci.1464-13.2013

Veit J, Bhattacharyya A, Kretz R, Rainer G (2011) Neural response dynamics of spiking and local field potential activity depend on CRT monitor refresh rate in the tree shrew primary visual cortex. J Neurophysiol 106:2303-2313. doi:10.1152/jn.00388.2011

Violet JM, Downie DL, Nakisa RC, Lieb WR, Franks NP (1997) Differential sensitivities of mammalian neuronal and muscle nicotinic acetylcholine receptors to general anesthetics. Anesthesiology 86:866-874. doi:10.1097/00000542-19970400000017

Worgotter F, Suder K, Zhao YQ, Kerscher N, Eysel UT, Funke K (1998) State-dependent receptive-field restructuring in the visual cortex. Nature 396:165-168. doi:10.1038/24157

Xing D, Yeh CI, Burns S, Shapley RM (2012) Laminar analysis of visually evoked activity in the primary visual cortex. Proc Natl Acad Sci USA 109:13871-13876. doi:10.1073/pnas.1201478109

Zhu YJ, Qiao WH, Liu KF, Zhong HY, Yao HS (2015) Control of response reliability by parvalbumin-expressing interneurons in visual cortex. Nat Commun. doi:10.1038/Ncomms7802

Zohary E, Shadlen MN, Newsome WT (1994) Correlated neuronal discharge rate and its implications for psychophysical performance. Nature 370:140-143. doi:10.1038/370140a0 